\title{
A CASE OF MANIFESTING CARRIER WITH DMD PHENOTYPE
}

\author{
Akshay Anand, Monika Vinish, Sudesh Prabhakar \\ Departments of Neurology, Postgraduate Institute of Medical Education and Research (PGIMER), Sector-12, Chandigarh, India
}

Summary: A case of a 35-year old female with a history of proximal weakness in lower limbs and bulkiness of both calves manifesting before ten years of age was reported. Clinical findings were suggestive of muscular dystrophy. Genetic analysis using polymerase chain reaction (PCR), single strand conformation polymorphism (SSCP) and direct sequencing revealed several point mutations, which account for dystrophin dysfunction and DMD type pathogenesis.

Key words: Dystrophin; PCR; Manifest carrier; DMD; Point mutations

\section{Introduction}

Duchenne and Becker muscular dystrophies (DMD/ BMD) are allelic, X-linked, recessive neuromuscular disorders caused by mutations in the dystrophin gene located at Xp21.1. Due to the X-linked nature of the disorder, males carrying the mutated gene are spontaneously affected, while females become carriers of the disease. DMD is a common disease affecting 1/3500 male births, while BMD is milder and less frequent. Diagnosis of patients with DMD/ BMD is usually definitive based on clinical, pathological and biochemical findings, although it is increasingly being confirmed by molecular analysis. Due to the extremely large size of the gene $(2.4 \mathrm{Mb})$, the majority of the mutations have been identified as deletions $(65 \%)$ or duplications $(5 \%)(5,7)$. The deletions in the dystrophin gene are non-randomly distributed in two regions, at the $5^{\prime}$ terminus (minor "hotspot") and in the distal half of the central rod domain around exons 44-53 (major "hotspot") $(3,7)$. Mutations that disrupt the open reading frame (ORF) are known to cause DMD, whereas those which maintain it have been suggested to result in BMD. It presents with muscular weakness, hypertrophy of the calf muscles, positive Gower's sign, and Pradhan's valley sign. We report, a female presenting with weakness in the lower limbs and bulkiness of both calves who was admitted to the neurology ward, PGIMER, Chandigarh. She presented with a positive family history including an affected son who was diagnosed as having DMD. Genetic analysis of dystrophin loci of this female and her son was conducted. PCR-SSCP and sequencing analysis were also performed to study the role of genetic polymorphism. Results revealed point mutations in the dystrophin gene in the carrier and her son, strongly indicating that the female is a manifesting DMD carrier.

\section{Case report}

A 35-year old lady was admitted with a history suggestive of insidious onset, proximal weakness in the lower limbs and bulkiness of both calves since ten years of age. The weakness progressed with gradual involvement of truncal muscles and later proximal muscles in the upper limbs over the next 8-10 years. She denied any history of neck or facial weakness, dysphagia, ptosis or diplopia, stiffness, wasting, twitchings, contractures, cramps or exercise intolerance, sensory, urinary, respiratory or cardiac symptoms. There was no history suggestive of diabetes, hypothyroidism or chronic drug intake. Her birth or developmental history was not available. Family history revealed that her elder brother suffered from a similar weakness and bulkiness of calves starting in early childhood. His symptoms had gradually progressed to a bed bound state causing death by 17 years of age. Her 9 year old younger son also exhibited bulky calves and has developed similar symptoms for the last 4-5 years. A detail of their older generations was not available. Her neurological examination revealed bulky calves with a rubbery feel on palpation, positive valley sign, neck flexor muscle weakness, grade $3 / 5$ (MRC grading) $4+/ 5$ power proximally and distally in all the limbs coupled with hyporeflexia in the upper limbs and normal reflexes in the lower limbs. There were no contractures and mentation cranial nerve; sensory, cerebellar and cardiac examinations were normal. Her hemogram, biochemistry (including fasting blood sugar, renal parameters and serum electrolytes), electrocardiogram and ECHO were normal. Serum CPK couldn't be done since the patient had undergone FNAC procedure from calves 4 days prior to admission. Electromyography (EMG) revealed myopathic changes. Her muscle biopsy was suggestive of mild muscular dystrophy. Clinically, her son revealed bulky calves, positive valley sign 


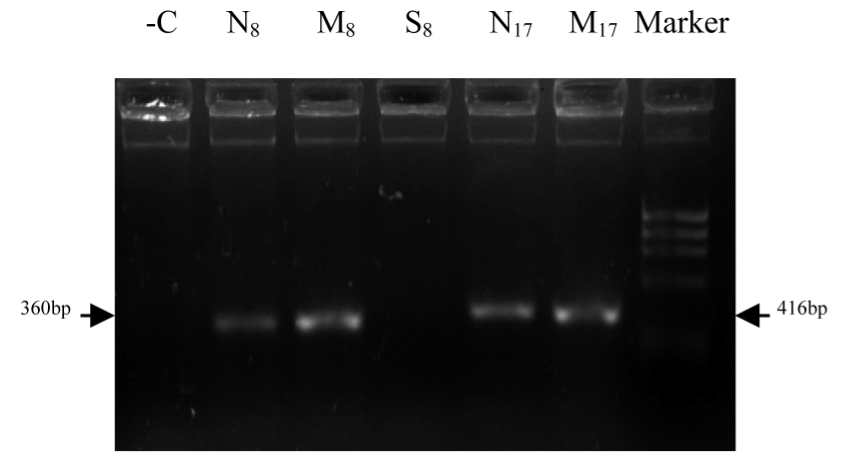

Fig. 1: PCR amplified products analysed on $2 \%$ agarose gel electrophoresis showing $360 \mathrm{bp}$ and 416 of exon 8 and exon 17 of Dystrophin gene in Normal (N), Mother (M) and Son (S).

-C - Negative control; N8 - Amplified PCR product of Exon 8 at 360 bp in normal subject; M8 - Amplified PCR product of Exon 8 at 360 bp in Mother; S8 - Absence of PCR product of Exon 8 in son; N17 - Amplified PCR product of Exon 17 at 416 bp in normal subject; M17 - Amplified PCR product of Exon 17at 416 bp in Mother; Marker - puc mix DNA marker

$\begin{array}{lllllllll}\mathrm{N}_{45} & \mathrm{M}_{45} & \mathrm{~S}_{45} & \mathrm{~N}_{48} & \mathrm{M}_{48} & \mathrm{~S}_{48} & \mathrm{~N}_{12} & \text { Marker }\end{array}$

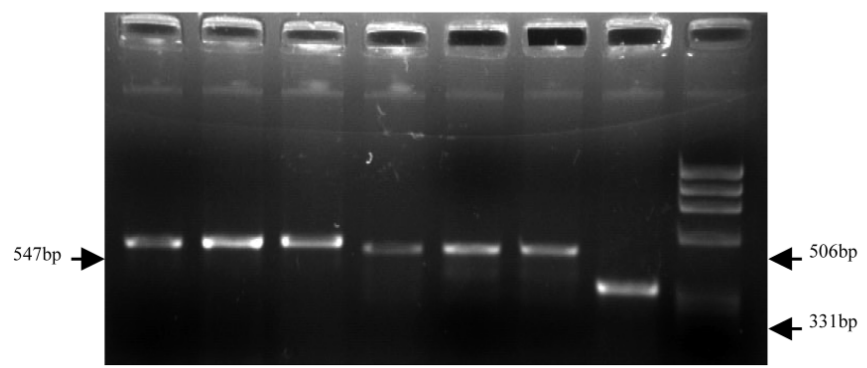

Fig. 3: PCR amplified products analysed on $2 \%$ Agarose gel electrophoresis showing $547 \mathrm{bp}, 506 \mathrm{bp}$ and $331 \mathrm{bp}$ of exon 45, 48 and exon 12 of Dystrophin gene in Normal $(\mathrm{N})$, Mother (M) and Son (S).

N45 - Amplified PCR product of Exon 45 at 547 bp in normal subject; M45 - Amplified PCR product of Exon 45 at 547 bp in Mother; S45 - Amplified PCR product of Exon 45 at 547 bp in son; N48 - Amplified PCR product of Exon 8 at 506 bp in normal subject; M48 - Amplified PCR product of Exon 4 at 506 bp in Mother; S48 - Amplified PCR product of Exon 45 at 506 bp in son; N12 - Amplified PCR product of Exon 45 at 331 bp in normal subject; Marker puc mix DNA marker

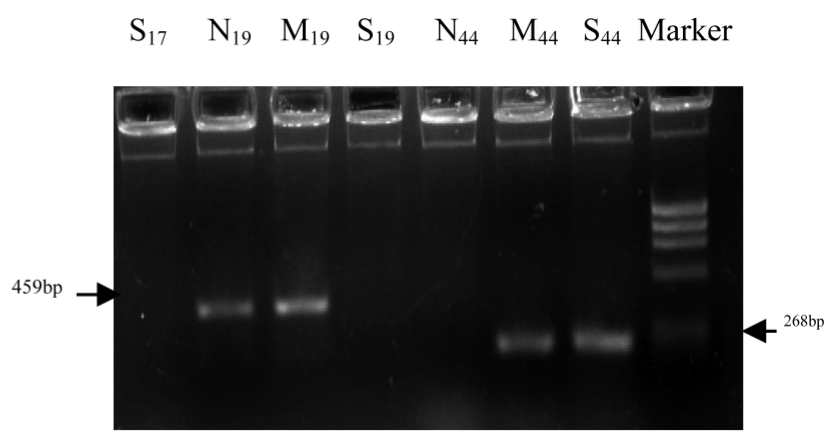

Fig. 2: PCR amplified products analysed on $2 \%$ Agarose gel electrophoresis showing $459 \mathrm{bp}$ and $268 \mathrm{bp}$ of exon 19 and exon 44 of Dystrophin gene in $\operatorname{Normal}(\mathrm{N})$, Mother (M) and Son $(\mathrm{S})$.

S17 - Absence of PCR product of Exon 17 in son; N19 Amplified PCR product of Exon 19 at 459 bp in normal subject; M19 - Amplified PCR product of Exon 19 at 459 bp in Mother; S19 - Amplified PCR product of Exon 19 at 459 bp in son; N44 - Absence of PCR product of Exon 8 in normal subject; M44 - Amplified PCR product of Exon 4 at 268 bp in Mother; Marker - puc mix DNA marker

$$
\begin{array}{llllllll}
\mathrm{M}_{12} & \mathrm{~S}_{12} & \mathrm{~N}_{51} & \mathrm{M}_{51} & \mathrm{~S}_{51} & \mathrm{~N}_{4} & \mathrm{M}_{4} & \mathrm{~S}_{4}
\end{array}
$$

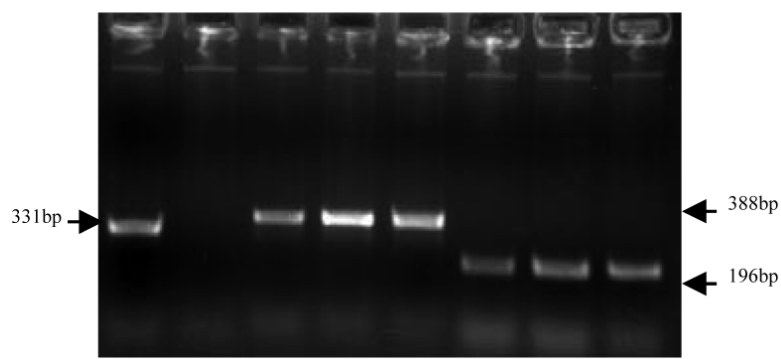

Fig. 4: PCR amplified products analysed on $2 \%$ Agarose gel electrophoresis showing $331 \mathrm{bp}, 388 \mathrm{bp}$ and 196bp of exon 12, exon 51 and exon 4 of Dystrophin gene in Normal $(\mathrm{N})$, Mother (M) and Son (S).

M12 - Amplified PCR product of Exon 12 at 331 bp in Mother; S12 - Absence of PCR product of Exon 12 in son; N51 - Amplified PCR product of Exon 51 at 388 bp in normal subject; M51 - Amplified PCR product of Exon 51 at 388 bp in Mother; S51 - Amplified PCR product of Exon 51 at 388 bp in son; N4 - Amplified PCR product of Exon 4 at 196 bp in normal subject; M4 - Amplified PCR product of Exon 4 at 196 bp in Mother; S4 - Amplified PCR product of Exon 4 at $196 \mathrm{bp}$ in son 
Tab. 1: Distribution of mutations in mother and son.

\begin{tabular}{|c|c|c|c|c|}
\hline Exon & Ex 4 & Ex 44 & Ex 45 & Exons deleted \\
\hline Mother & $\mathrm{G} \rightarrow \mathrm{A}$ & ‘a'- insertion 6561bp & g' insertion 6572 bp & None \\
& $\mathrm{G} \rightarrow \mathrm{C}$ & (Frame shift mutation) & (frame shift mutation) & \\
\hline Son & $\mathrm{G} \rightarrow \mathrm{A}$ & $\begin{array}{c}\text { ‘a’ del 6355 bp } \\
\text { (Frame shift mutation) }\end{array}$ & & $8,12,17,19$ \\
\hline
\end{tabular}

and Gower's maneuver, neck muscle and proximal muscle in all four limbs. Her serum CPK was $201 \mathrm{u} / \mathrm{L}$ (Normal 25-192 u/L), ECG was normal, EMG revealed myopathic changes and muscle biopsy was suggestive of muscular dystrophy.

Hence, the clinical possibility of dystrophinopathy in view of the pattern of inheritance became a consideration for the son and manifest carrier for the mother

Genomic DNA from the peripheral blood lymphocytes was isolated using the established phenol chloroform isolation method (12). It was quantitated and analysed for its integrity in $2 \%$ agarose gel electrophoresis. DNA of both mother and son was amplified for specific dystrophin gene by using a set of primers reported earlier (1), followed by SSCP and direct sequencing of the purified PCR products. The sequences obtained from these DMD patients were compared with sequences from the normal individuals. These were compared with the published sequences from pubmed and other gene bank databases.

PCR analysis has revealed the absence of exons 8, 12, 17, 19 , in the son while all the exons were intact in the mother (Fig. 1-4). When the amplified PCR products were subjected to SSCP, they exhibited significant differential mobility indicating heterozygous mutations. On sequencing, the PCR products of both mother and son revealed a number of point mutations (Tab. 1); some transitions were common in exon 4.

\section{Discussion}

Molecular genetics approaches have made it possible to establish a precise diagnosis and female carrier detection (10). However, carrier analysis by molecular methods in females is still difficult due to the presence of a normal copy of the gene in the hetrerozygous carrier females (9). Duchenne muscular dystrophy, being an X-linked recessive disorder rarely affects females, who usually act as non manifesting carriers. In this case, a 35-year-old female with clinically expressing DMD phenotype is reported In the present report, a combination of PCR, SSCP and sequencing was utilized to screen the mutations in the dystrophin loci and revealed that the mother exhibited frame shift mutation in the dystrophin gene, while her son exhibited both exonic deletions as well as point mutations in the same loci (Tab. 1). DMD manifest carrier is a rare case and this report represents the first clinical-molecular description from South-East Asia. (11) These "isolated carriers" are reported to represent about $10 \%$ of women with high serum creati- ne phosphokinase (CPK) levels with evident muscle involvement.

Several factors responsible for muscular dystrophy in a manifest female carrier include the presence of mutations in both alleles of Xp21.1. This also includes the possibility of Turner's syndrome generating such a clinical picture. However, features representative of this were found to be completely absent in this case. The shielding effect exerted through the normal $\mathrm{X}$ allele appears to be lost in such manifesting carriers as this. Different approaches have been used for the identification of female carriers where deletions are not detectable by PCR. These include multiple ligation probe amplification (MLPA) for the identification of female carriers of DMD deletions or duplications (4). Point mutations detected by SSCP and PCR, particularly in exon 44 and 45 in both mother and son, seem to have altered the protein structure. In the present case the mutations were detected in the ORF, which might be responsible for the pathogenesis this case. Similarly the presence of common transition mutations in exon 4 of both mother and son, some novel point mutations, might be causal of DMD phenotype.

The severity of the disease is well known to be causally linked with the type and severity of mutations. It has earlier been reported that about $60 \%$ of both Duchenne muscular dystrophy (DMD) and Becker muscular dystrophy (BMD) are caused due to deletions of the dystrophin gene (6). The „reading frame" hypothesis predicts that BMD patients produce a semifunctional, internally deleted dystrophin protein, whereas DMD patients produce a severely truncated protein that is unstable. Alternate splicing can also produce an unpredictable coding sequence as reported in earlier studies (2). Thus, we can speculate that these point mutations in the mother might be responsible for alterations of the reading frame and hence the DMD type phenotype.

\section{References}

1. Chamberlain JS, Gibbs RA, Ranier JE, Caskey CT Deletion screening of the Duchenne and Becker muscular dystrophy locus via multiplex DNA amplification. Nucleic Acid Res 1988;16:11141-56.

2. Chelly J, Gilgenkrantz H, Lambert H, et al. Effect of dystrophin gene deletions on mRNA levels and processing in Duchenne and Becker muscular dystrophy. Cell 1990;63:1239-48

3. Forrest SM, Cross GS, Flint T, et al. Further studies of gene deletions that cause Duchenne and Becker muscular dystrophies. Genomics 1988;2:109-14.

4. Gatta V, Scarciolla O, Gaspari AR, et al. Identification of deletions and duplications of the DMD gene in affected males and carrier females by multiple ligation probe amplification (MLPA). Hum Genet. $2005 ; 117(1): 92-8$.

5. Hoffman EP, Kunkel LM, Dystrophin abnormalities in Duchenne/Becker muscular dystrophy. Neuron 1989;2:1019-29. 
6. Koenig M, Beggs AH, Moyer M. et al. The molecular basis for Duchenne versus Becker muscular dystrophy: correlation of severity with type of deletion. Am J Hum Genet 1989:45:498-506.

7. Koenig M, Hoffman EP, Bertelson CJ, et al. Complete cloning of the Duchenn muscular dystrophy (DMD) cDNA and preliminary genomic organisation of the DMD gene in normal and affected individuals. Cell 1987;50:509-17.

8. Monaco AP, Bertelson CJ, Liechti-Gallati S, et al. An explanation for the phenotypic differences between patients bearing partial deletions of the DMD locus. Genomics 1988; 2:90-5.
9. Mukherjee M, Mittal B. Muscular dystrophies. Indian J Pediatr 2004;71:161-8. 10. Nevo Y, Shomrat R, Yaron Y, Orr-Urtreger A, Harel S, Legum C. Fetal muscle biopsy as a diagnostic tool in Duchenne muscular dystrophy. Prenat Diagn 1999;19(10):921-6

11. Padma MV, Jain S, Sarkar C, Maheshwari MC. Isolated manifesting carrier of Duchenne muscular dystrophy. Neurology India. 1995; 43(1): 42-4

12. Sambrook J, Fritsch EF, Maniattis T. In: Molecular cloning - a laboratory manual, Vol. 1-3 Cold spring harbor laboratory Press, New York, Third edition. Eds. Nancy Ford, Chris Nolan and Michele Ferguson (2001).

Received: $15 / 03 / 2009$.

Accepted in revised form: 23/10/2009.

\section{Corresponding author:}

Sudesh Prabhakar, Department of Neurology, Post Graduate Institute of Medical Education and Research, Chandigarh, India; e-mail: pgineurology@gmail.com 